\title{
Análisis de Von Neumann para el método Local Discontinuous Galerkin en 1D
}

\author{
Paul Castillo $^{a *}$, Sergio Gómez ${ }^{b}$ \\ a Universidad de Puerto Rico, Departamento de Ciencias Matemáticas, Mayagüez, \\ Puerto Rico. \\ $b$ Universidad Nacional Autónoma de Honduras, Escuela de Matemática y Ciencias \\ de la Computación, D.C. Tegucigalpa, Honduras.
}

Resumen. Utilizando el análisis de von Neumann como herramienta teórica, se desarrolla un análisis sobre las condiciones de estabilidad de algunos métodos explícitos de avance en tiempo, en combinación con la discretización espacial Local Discontinuous Galerkin (LDG) por sus siglas en inglés y aproximaciones de alto orden. La constante de estabilidad CFL (CourantFriedrichs-Lewy) se estudia en función de los parámetros del método LDG y el grado de aproximación. Se realiza una serie de experimentos numéricos para validar los resultados teóricos.

Palabras clave: análisis de estabilidad de Von Neumann, CFL, Local Discontinuous Galerkin (LDG).

MSC2010: 65M12, 65M20, 65M60.

\section{Von Neumann analysis for the Local Discontinuous Galerkin method in $1 D$}

\begin{abstract}
Using the von Neumann analysis as a theoretical tool, an analysis of the stability conditions of some explicit time marching schemes, in combination with the spatial discretization Local Discontinuous Galerkin (LDG) and high order approximations, is presented. The stability constant, CFL (Courant-Friedrichs-Lewy), is studied as a function of the LDG parameters and the approximation degree. A series of numerical experiments is carried out to validate the theoretical results.

Keywords: Von Neumann stability analysis, CFL, Local Discontinuous Galerkin (LDG).

\footnotetext{
*E-mail: paul.castillo@upr.edu

Recibido: 7 de noviembre de 2018, Aceptado: 16 de abril de 2019.

Para citar este artículo: P. Castillo, S. Gómez, Análisis de Von Neumann para el método Local Discontinuous

Galerkin en 1D, Rev. Integr. temas mat. 37 (2019), No. 2, 199-217. doi: 10.18273/revint.v37n2-2019001.
} 


\section{Introducción}

Uno de los conceptos fundamentales en la simulación de problemas transitorios es el de estabilidad numérica. En términos simples, este establece la relación que debe ocurrir entre la resolución temporal y la espacial de un esquema numérico a fin de que la aproximación no presente oscilaciones, las cuales podrían crecer sin control, degradando completamente la calidad de la aproximación. Esta noción es de gran interés, en particular al aplicar discretizaciones temporales explícitas.

En las últimas décadas los métodos de elemento finito de Galerkin discontinuos han sido ampliamente utilizados en una extensa variedad de ecuaciones. A diferencia del método de elemento finito clásico (continuo), los métodos discontinuos se caracterizan fundamentalmente por imponer continuidad en forma débil, por lo que la aproximación no requiere continuidad entre celdas. Esto posibilita su formulación variacional, de manera natural, en mallas suficientemente arbitrarias, como por ejemplo aquellas que poseen nodos colgantes. Además, permite el uso de aproximaciones polinomiales de grado arbitrario, pero no necesariamente uniforme, lo cual es ideal para la versión $h p$ del método de elemento finito. Aquí se considerará uno de estos métodos, conocido en inglés como Local Discontinuous Galerkin (LDG), el cual fue propuesto y analizado por Cockburn y Shu en [8], para problemas transitorios de difusión y convección no lineal.

Este artículo tiene como objetivo derivar algunas condiciones necesarias y suficientes para la estabilidad numérica del método LDG, aplicado a ecuaciones clásicas basadas en el operador laplaciano, en combinación con esquemas explícitos en tiempo. Si bien es cierto que para problemas de difusión el uso de discretizaciones temporales implícitas es lo más recomendable, algunos investigadores prefieren, por su sencillez en la programación, el uso de métodos explícitos en tiempo, los cuales no requieren resolver sistemas lineales o no lineales. Por lo que es importante hacer un análisis de las condiciones de estabilidad; o de la constante de estabilidad CFL (Courant-Friedrichs-Lewy), en función de los parámetros característicos del método y del grado de aproximación. Con este objetivo en mente, se repasa brevemente el concepto de estabilidad numérica y se introduce una de las herramientas teóricas esenciales: el análisis de estabilidad de Von Neumann, también conocido como análisis de estabilidad de Fourier. Aunque nuestro análisis se restringe al caso unidimensional, el uso de dicha herramienta para el estudio de la constante CFL en un método Galerkin discontinuo no es del todo trivial, ya que su aplicación a una sola ecuación es similar a la de analizar la condición CFL para un sistema de ecuaciones.

El estudio de la CFL para discretizaciones espaciales de tipo Galerkin discontinuo ha sido considerado mayormente en el caso de problemas hiperbólicos, donde las discretizaciones temporales explícitas son preferiblemente utilizadas. Por ejemplo, Kubatko, Westering y Dawson [11] presentaron un estudio numérico de la condición CFL para una clase de métodos de Runge Kutta explícitos, aplicada a un problema de advección lineal unidimensional; y posteriormente, dicho estudio fue extendido, por los mismos autores, a mallas triangulares en [10], y por Toulorge y Desmet en [14]. Recientemente, Castillo y Gómez [4] estudiaron la condición CFL del método LDG aplicado a la ecuación fraccionaria nolineal de Schrödinger, utilizando el método "pídola" (conocido en inglés como Leapfrog) como esquema de avance de tiempo. En dicho estudio se concluye que el uso de métodos explícitos puede ser una muy buena alternativa cuando el orden de la difusión fraccionaria es cercano a 1.

[Revista Integración, temas de matemáticas 
La organización de este artículo es la siguiente. En la Sección 2 se introduce el concepto de estabilidad numérica. Aunque este ha sido mayormente utilizado en el contexto de discretizaciones basadas en diferencias finitas, también puede ser aplicado al método de elemento finito clásico en mallas cartesianas uniformes; y por consiguiente, también para los métodos de tipo Galerkin discontinuo, que son los de nuestro interés. En la Sección 3 se presenta la idea general de la discretización del operador laplaciano por medio del método LDG, utilizando como ecuación modelo la de calor en una dimensión. En la Sección 4, utilizando el análisis de estabilidad de von Neumann de la Sección 2.1, se analizan las condiciones de estabilidad numérica del método LDG aplicado a algunas ecuaciones clásicas, como la ecuación de calor, la ecuación lineal de Schrödinger y la de onda, para varias discretizaciones en tiempo.

\section{Estabilidad numérica}

Considérese el siguiente problema de valores iniciales: $u(x, 0)=u_{0}(x)$ para todo $x \in \mathbb{R}$,

$$
\mathcal{P}(u)=\partial_{t} u-\mathcal{L}(u)=0, \quad(t, x) \in \mathbb{R} \times(0, T],
$$

donde $\partial_{t} u$ denota la derivada parcial con respecto a $t ; \mathcal{L}(\cdot)$ es un operador diferencial lineal en la variable espacial $x$. Se denotan por $h, \tau \in \mathbb{R}^{+}$los parámetros característicos de la discretización en espacio y tiempo, respectivamente; y por $u_{m}^{n}$ la aproximación de $u(m h, n \tau)$ para cualesquiera $m \in \mathbb{Z}$ y $n \in \mathbb{N}$. La noción de estabilidad numérica sintetiza la idea de que en cualquier tiempo, $t_{n}=n \tau<T$, la norma de la aproximación en ese tiempo está acotada uniformemente con respecto a $\tau$ y $h$ a medida que estos tienden a cero. Este concepto se formaliza de la siguiente manera: sea $l_{2}(h \mathbb{Z})$ el espacio de las sucesiones de valor complejo $u=\left(u_{m}\right)_{m \in \mathbb{Z}}$ tales que $\sum_{m \in \mathbb{Z}}\left|u_{m}\right|^{2}<\infty$, dotado, por conveniencia, de la norma, dependiente de $h$, definida por

$$
\|u\|_{h}^{2}=h \sum_{m \in \mathbb{Z}}\left|u_{m}\right|^{2}, \quad \forall u \in l_{2}(h \mathbb{Z}) .
$$

Se considera la siguiente definición de estabilidad numérica utilizada en los libros de Strickwerda [13] y Gustafsson, Kreiss y Oliger [9]:

Definición 2.1 (Estabilidad numérica). Se dice que un esquema $\mathcal{P}_{\tau, h}$ para el problema $\mathcal{P}$ es estable en una región $\Gamma$, si existe un entero $J$ tal que para todo tiempo $T>0$ existe una constante $C_{T}$ tal que para todo $0 \leq n \tau \leq T$ con $(h, \tau) \in \Gamma$ se verifique

$$
\left\|u^{n}\right\|_{h} \leq C_{T} \sum_{j=0}^{J}\left\|u^{j}\right\|_{h} .
$$

Nótese que la cota superior solamente depende de la norma de un número fijo, $J$, de aproximaciones iniciales; y que la constante $C_{T}$ depende del tiempo final, pero no de los tiempos intermedios ni de los parámetros $\tau$ y $h$.

\subsection{Análisis de von Neumann}

Por lo general, la aplicación de la Definición 2.1 a esquemas numéricos de alta precisión requiere cálculos muy engorrosos. En [5], haciendo uso de la transformada discreta de

Vol. 37, $\left.\mathrm{N}^{\circ} 2,2019\right]$ 
Fourier, Charney, Fjörtoft y von Neumann desarrollaron, rigurosamente, una herramienta para determinar las condiciones de estabilidad de un esquema numérico, la cual se conoce como análisis de estabilidad de von Neumann o análisis de estabilidad local de Fourier. La idea fundamental es motivada por i) la igualdad de Parseval, (ver más adelante la Proposición 2.3), la cual establece que la transformada discreta de Fourier es una isometría y, ii) el hecho de que en el espacio de frecuencias la norma de la aproximación en el tiempo $t_{n+1}$ se puede expresar como la norma de la aproximación en el tiempo anterior, $t_{n}$, multiplicada por un factor de amplificación. Es importante mencionar que esta herramienta posee la limitación de restringirse a problemas lineales con coeficientes constantes, condiciones periódicas o en un dominio infinito y discretizaciones en mallas uniformes. No obstante, a pesar de dichas restricciones, se puede obtener una idea sobre la estabilidad de un esquema en el caso de problemas nolineales, al aplicar este análisis a una linearización del problema original.

Sea $L_{2}[-\pi / h, \pi / h]$ el espacio de funciones medibles, $f:[-\pi / h, \pi / h] \longrightarrow \mathbb{C}$ tal que $\|f\|^{2}=\int_{-\pi / h}^{\pi / h}|f|^{2}<\infty$, donde la integración se realiza en el sentido de Lebesgue. La transformada de Fourier y su inversa se definen como sigue:

Definición 2.2. La transformada discreta de Fourier, $\mathcal{F}_{h}$, y su inversa, $\mathcal{F}_{h}^{-1}$, son operadores lineales definidos por

a) $\mathcal{F}_{h}: l_{2}(h \mathbb{Z}) \longrightarrow L_{2}\left[-\frac{\pi}{h}, \frac{\pi}{h}\right], u \longmapsto \hat{u}$, tal que para todo $\omega \in\left[-\frac{\pi}{h}, \frac{\pi}{h}\right]$,

$$
\hat{u}(\omega)=\frac{h}{\sqrt{2 \pi}} \sum_{m \in \mathbb{Z}} e^{-i m h \omega} u_{m} ;
$$

b) $\mathcal{F}_{h}^{-1}: L_{2}\left[-\frac{\pi}{h}, \frac{\pi}{h}\right] \longrightarrow l_{2}(h \mathbb{Z}), \widehat{u} \longmapsto u$ tal que para todo $m \in \mathbb{Z}$,

$$
u_{m}=\frac{1}{\sqrt{2 \pi}} \int_{-\frac{\pi}{h}}^{\frac{\pi}{h}} e^{i m h \omega} \widehat{u}(\omega) d \omega .
$$

El uso de esta transformada discreta es motivado por la igualdad de Parseval, la cual permite expresar la Definición 2.1 en el espacio de frecuencia, preservando las normas.

Proposición 2.3 (Igualdad de Parseval). Para todo $u \in l_{2}(h \mathbb{Z})$, sea $\widehat{u}=\mathcal{F}(u)$; entonces se tiene

$$
\|\widehat{u}\|^{2}=\|u\|_{h}^{2}
$$

Como breve repaso, a continuación, se ilustra el uso de la transformada discreta de Fourier (análisis de von Neumann) para el análisis de las condiciones de estabilidad numérica del siguiente esquema explícito, conocido como FTCS, por sus siglas en inglés (Forward in Time Central in Space):

$$
\frac{u_{m}^{n+1}-u_{m}^{n}}{\tau}=\sigma \frac{u_{m-1}^{n}-2 u_{m}^{n}+u_{m+1}^{n}}{h^{2}},
$$

el cual aproxima la solución de la ecuación del calor,

$$
\partial_{t} u-\sigma \partial_{x x} u=0, \sigma>0 .
$$

[Revista Integración, temas de matemáticas 
Este esquema se deriva considerando en $\left(x_{m}, t_{n}\right)=(m h, n \tau)$ la aproximación por diferencias hacia adelante para la derivada temporal, y la central, para la derivada espacial. Aplicando la inversa de la transformada discreta de Fourier, a cada término del esquema (4), y por la unicidad de la transformada, se deduce la relación

$$
\widehat{u}^{n+1}=\left(\sigma \mu e^{-i h \omega}+(1-2 \sigma \mu)+\sigma \mu e^{i h \omega}\right) \widehat{u}^{n}=g(h \omega) \widehat{u}^{n}
$$

donde $\mu=\frac{\tau}{h^{2}}$. Esta ecuación indica que en el espacio de frecuencias $\widehat{u}^{n+1}$ se obtiene multiplicando $\widehat{u}^{n}$ por el factor de amplificación o símbolo $g(h \omega)=1-4 \sigma \mu \sin ^{2}\left(\frac{1}{2} h \omega\right)$. Charney y col. [5] observaron que la estabilidad de un esquema se reduce al estudio de este factor. Para este ejemplo una condición necesaria y suficiente para garantizar la estabilidad del esquema numérico es

$$
|g(h \omega)| \leq 1, \quad \forall h \omega \in[-\pi, \pi]
$$

De manera análoga se puede considerar el método explícito de Euler (4) para la ecuación lineal de Schrödinger

$$
\partial_{t} u-i \sigma \partial_{x x} u=0, \quad \sigma>0
$$

Aplicando el análisis de estabilidad de von Neumann se obtiene el factor de amplificación

$$
g(h \omega)=1-i 4 \sigma \mu \sin ^{2}\left(\frac{1}{2} h \omega\right),
$$

del cual se deduce que, para todo $h \omega \in[-\pi, \pi]$,

$$
|g(h \omega)|^{2}=1+16 \sigma^{2} \mu^{2} \sin ^{4}\left(\frac{1}{2} h \omega\right),
$$

por lo que este esquema es incondicionalmente inestable.

La transformada discreta de Fourier también puede definirse en el espacio de sucesiones $u=\left(\boldsymbol{u}_{m}\right)_{m \in \mathbb{Z}}$, donde $\boldsymbol{u}_{m} \in \mathbb{C}^{d}$ para cualesquiera $m \in \mathbb{Z}$ y $d \in \mathbb{N}$. Lo cual permite extender el análisis de von Neumann a esquemas numéricos aplicados a sistemas de ecuaciones. En este caso las condiciones de estabilidad son más delicadas de obtener, ya que el factor de amplificación es una matriz, $G(\omega, h, \tau)$. El criterio de estabilidad análogo a la definición 2.1, en el espacio de frecuencias, se traduce de la siguiente manera: para todo $T>0$ existe una constante $C_{T}$ (la cual solamente depende de $T$ ), para la cual

$$
\left\|G(\omega, h, \tau)^{n}\right\| \leq C_{T}, \quad 0 \leq n \tau \leq T, \omega \in[-\pi, \pi], 0<h<h_{o} .
$$

En algunos casos, el análisis de estabilidad se reduce al estudio de la relación entre $h$ y $\tau$ de tal forma que se verifique la llamada Condición de von Neumann, $\rho(G) \leq 1+\mathcal{O}(\tau)$, o su versión más estricta $\rho(G) \leq 1$. Sin embargo, puesto que para toda matriz $A \in \mathbb{C}^{d \times d}$ y para todo $n \in \mathbb{N}$ se tiene la desigualdad $\rho^{n}(A) \leq\left\|A^{n}\right\|$, la cual puede ser estricta, la condición de von Neumann es una condición de estabilidad necesaria pero no suficiente. Algunos resultados que garantizan que la condición de von Neumann es además suficiente se demuestran en $[9,13]$ y sus referencias.

Vol. 37, $\left.\mathrm{N}^{\circ} 2,2019\right]$ 


\section{Método LDG}

La formulación variacional del método LDG aplicado a un operador diferencial de segundo orden se ilustra a continuación, utilizando como modelo la ecuación de calor escrita como un sistema de ecuaciones de primer orden:

$$
\begin{aligned}
\sigma^{-1} \boldsymbol{q}+\partial_{x} u & =0 \\
\partial_{t} u+\partial_{x} \boldsymbol{q} & =0
\end{aligned}
$$

Sea $\mathcal{T}_{h}=\left\{x_{m}\right\}_{m \in \mathbb{Z}}$ una partición uniforme de $\mathbb{R}, \mathrm{y} \psi_{m}:[-1,1] \longrightarrow I_{m}$ el mapa local de la celda $I_{m}=\left[x_{m}, x_{m+1}\right]$, para todo $m \in \mathbb{Z}$. Se denota por $\mathcal{L}=\left\{L_{j}\right\}_{j=0}^{p}$ la familia de polinomios ortogonales de Legendre, la cual forma una base en el espacio $\mathbb{P}_{p}([-1,1])$ de polinomios de grado menor o igual a $p$, restringidos a la celda de referencia $[-1,1]$; entonces, para todo $m \in \mathbb{Z}$, la familia $\mathcal{B}_{m}=\left\{\phi_{j}=L_{j} \circ \psi_{m}\right\}_{j=0}^{p}$ es una base polinomial del espacio de elemento finito $\mathbb{P}_{p}\left(I_{m}\right)$. En cada paso de tiempo $t \in(0, T]$, y para cada celda $I_{m}, m \in \mathbb{Z}$, el método LDG busca una aproximación de la forma

$$
u_{h}(x, t)=\sum_{j=0}^{p} U_{j}^{m}(t) \phi_{j}(x) \quad \text { y } \quad \boldsymbol{q}_{h}(x, t)=\sum_{j=0}^{p} Q_{j}^{m}(t) \phi_{j}(x),
$$

tales que las siguientes ecuaciones se cumplan, para todo $\boldsymbol{r}, v \in \mathbb{P}_{p}\left(I_{m}\right)$ :

$$
\begin{array}{r}
\int_{I_{m}} \sigma^{-1} \boldsymbol{r} \boldsymbol{q}_{h}+\left.\widehat{u}_{h} \boldsymbol{r}\right|_{x_{m}} ^{x_{m+1}}-\int_{I_{m}} u_{h} \partial_{x} \boldsymbol{r}=0, \\
\int_{I_{m}} v \partial_{t} u_{h}+\left.v \widehat{\boldsymbol{q}_{h}}\right|_{x_{m}} ^{x_{m+1}}+\left.v s\left(u_{h}\right)\right|_{x_{m}} ^{x_{m+1}}-\int_{I_{m}} \boldsymbol{q}_{h} \partial_{x} v=0 .
\end{array}
$$

Los flujos numéricos $\widehat{u}_{h}\left(x_{m}\right)$ y $\widehat{\boldsymbol{q}_{h}}\left(x_{m}\right)$ son aproximaciones de $u$ y $\boldsymbol{q}$, respectivamente, en cada nodo $x_{m}$ de la malla, y se definen como combinaciones convexas de los valores de $u_{h}$ y $\boldsymbol{q}_{h}$ por ambos lados del nodo:

$$
\begin{aligned}
& \widehat{u}_{h}\left(x_{m}\right)=\left(1-\zeta_{m}\right) u_{h}\left(x_{m}^{-}\right)+\zeta_{m} u_{h}\left(x_{m}^{+}\right), \\
& \widehat{\boldsymbol{q}_{h}}\left(x_{m}\right)=\zeta_{m} \boldsymbol{q}_{h}\left(x_{m}^{-}\right)+\left(1-\zeta_{m}\right) \boldsymbol{q}_{h}\left(x_{m}^{+}\right),
\end{aligned}
$$

donde $\zeta_{m} \in[0,1]$. Esta definición particular preserva la simetría de la discretización del operador diferencial de segundo orden, $\mathcal{L}(u)=-u^{\prime \prime}$ (para más detalles, ver [2]). En este trabajo se consideran tres casos particulares de flujos:

1) Izquierdo $\left(\zeta_{L}\right): \zeta_{m}=1$ para todo $m$;

2) Central $\left(\zeta_{C}\right): \zeta_{m}=1 / 2$ para todo $m$;

3) Derecho $\left(\zeta_{R}\right): \zeta_{m}=0$ para todo $m$.

La selección de los flujos influye únicamente en la densidad del operador discreto, pero no en el orden de convergencia del método. En [1] Castillo propuso algunas heurísticas para reducir el código de plantilla del operador discreto para problemas en 2D y 3D. En

[Revista Integración, temas de matemáticas 
problemas unidimensionales, los flujos direccionales (izquierdo y derecho) producen un código compacto, mientras que el central produce uno de mayor tamaño.

En [2] se presentó, por primera vez, un análisis de error para la versión mixta del método en el régimen estacionario y dominios en $2 \mathrm{D}$. Se demostró que las variables $u_{h}$ y $\boldsymbol{q}_{h}$ convergen, para cualquier flujo numérico, con orden $\mathcal{O}\left(h^{p+1}\right)$ y $\mathcal{O}\left(h^{p}\right)$, respectivamente. Perugia y Schötzau [12] realizaron un análisis de error para la variable primaria $u_{h} \mathrm{y}$ la versión $h p$. En ambos análisis, la existencia y unicidad de la solución del problema discreto se garantiza utilizando el término de estabilización $s\left(u_{h}\right)$ de la ec. (9b), definido por

$$
s\left(u_{h}\right)\left(x_{m}\right)=\eta_{m}\left(u_{h}\left(x_{m}^{-}\right)-u\left(x_{m}^{+}\right)\right), \quad \eta_{m}>0 .
$$

Sin embargo, Cockburn y Dong [7], utilizando flujos direccionales, mostraron estabilidad del método aun cuando $\eta=0$ en todas las aristas interiores. Para diferenciar esta nueva versión de la clásica o estabilizada $(\eta>0)$, esta se conoce como LDG de disipación mínima (md-LDG). El análisis del método en dominios unidimensionales y problemas lineales transitorios de convección y difusión fue presentada en [3] para la versión $h p$, donde además se obtuvo convergencia para aproximaciones constantes, es decir $p=0$. El caso multidimensional fue analizado por Cockburn y Dawson en [6].

Sean $\boldsymbol{q}_{m}=\left(Q_{j}^{m}\right)_{j=0}^{p}$ y $\boldsymbol{u}_{m}=\left(U_{j}^{m}\right)_{j=0}^{p}$ los vectores de coeficientes que representan $\boldsymbol{q}_{h}$ y $u_{h}$, respectivamente, en la base local $\mathcal{B}_{m}$ de la celda $I_{m}$. Suponiendo constante el parámetro de estabilidad $\eta$, la formulación semi-discreta (ecuaciones (9a) y (9b)) se escribe localmente como un sistema de ecuaciones diferenciales ordinarias de la siguiente forma:

$$
\begin{aligned}
\sigma^{-1} M_{m} \boldsymbol{q}_{m} & =B_{1}\left(\boldsymbol{u}_{m-1}, \boldsymbol{u}_{m}, \boldsymbol{u}_{m+1}\right) \\
M_{m} \frac{d \boldsymbol{u}_{m}}{d t} & =-B_{2}\left(\boldsymbol{q}_{m-1}, \boldsymbol{q}_{m}, \boldsymbol{q}_{m+1}\right)-\eta S\left(\boldsymbol{u}_{m-1}, \boldsymbol{u}_{m}, \boldsymbol{u}_{m+1}\right) .
\end{aligned}
$$

\section{Análisis de estabilidad.}

Contrariamente al método de diferencias finitas, la aplicación del análisis de von Neumann para discretizaciones espaciales discontinuas es, por lo general, más complicada, en particular para aproximaciones de alto orden, es decir, para $1 \leq p$. Esto se debe a que el factor de amplificación es una matriz. El caso $p=0$, aproximaciones constantes por celda, corresponde al método de volumen finito; su análisis es similar al de un esquema de diferencias finitas y el factor de amplificación es un escalar. Las condiciones de estabilidad necesarias, y en algunos casos suficientes, para el método LDG aplicado a un problema escalar, se obtienen del análisis de estabilidad de von Neumann en su versión de sistemas, mencionado en la Sección 2.1. Para poder llevar a cabo el análisis de estabilidad, se asume de aquí en adelante que el dominio está dividido en celdas de igual longitud $h$.

\subsection{Ecuación de calor}

Como primer ejemplo se analiza la condición CFL de la discretización espacial LDG aplicada a la ecuación de calor, ecuación (5), utilizando el método de Euler explícito

Vol. 37, $\left.\mathrm{N}^{\circ} 2,2019\right]$ 
para la discretización en tiempo. El método completamente discretizado, FT-LDG, toma la siguiente forma en la celda $I_{m}$ :

$$
\begin{aligned}
\boldsymbol{q}_{m}^{n+1} & =\sigma M_{m}^{-1} B_{1}\left(\boldsymbol{u}_{m-1}^{n}, \boldsymbol{u}_{m}^{n}, \boldsymbol{u}_{m+1}^{n}\right), \\
\boldsymbol{u}_{m}^{n+1} & =\boldsymbol{u}_{m}^{n}-\tau M_{m}^{-1} B_{2}\left(\boldsymbol{q}_{m-1}^{n+1}, \boldsymbol{q}_{m}^{n+1}, \boldsymbol{q}_{m+1}^{n+1}\right)-\tau \eta M_{m}^{-1} S\left(\boldsymbol{u}_{m-1}^{n}, \boldsymbol{u}_{m}^{n}, \boldsymbol{u}_{m+1}^{n}\right),
\end{aligned}
$$

donde el superíndice $n+1$ se refiere al tiempo discreto $t_{n+1}=t_{n}+\tau$ y $M_{m}$ es la matriz de masa local en la celda $I_{m}$.

Sean $\widehat{M}_{m}, \widehat{B}_{1}, \widehat{B}_{2}$ y $\widehat{S}$ los símbolos de los operadores $M_{m}(\cdot), B_{1}(\cdot), B_{2}(\cdot)$ y $S(\cdot)$, respectivamente, los cuales pueden depender de $h, \omega$ y $\tau$.

Proposición 4.1. El símbolo del operador laplaciano discreto, $\Delta_{h}(\cdot)$, generado por el método $L D G$ es

$$
\widehat{\Delta}_{h}=-\left(\widehat{B}_{1}^{*} M_{m}^{-1} \widehat{B}_{1}+\eta \widehat{S}\right) .
$$

Demostración. Aplicando la transformada discreta de Fourier, en su versión de sistemas, a la ecuación (12a) y al término de la derecha de la ecuación (12b), se obtiene

$$
\widehat{M}_{m} \widehat{\boldsymbol{q}_{h}}=\widehat{B}_{1} \widehat{u_{h}} \quad \text { y } \quad \widehat{\Delta}_{h} \widehat{u_{h}}=-\widehat{B}_{2} \widehat{\boldsymbol{q}_{h}}-\eta \widehat{S} \widehat{u_{h}} \quad \therefore \quad \widehat{\Delta}_{h}=-\left(\widehat{B}_{2} \widehat{M}_{m}^{-1} \widehat{B}_{1}+\eta \widehat{S}\right) .
$$

El resultado se obtiene observando que, por la definición de los flujos numéricos (10a) y (10b), se tiene la relación $\widehat{B}_{2}=\widehat{B}_{1}^{*}$, donde ${ }^{*}$ denota el operador adjunto (conjugada compleja de una matriz), y que $\widehat{M}_{m}=M_{m}$.

Por conveniencia para el análisis, el parámetro de estabilización $\eta$ se escribirá de la siguiente forma

$$
\eta=2 \frac{\gamma}{h}, \quad \gamma \geq 0
$$

Nótese que el valor $\gamma=0$ se refiere al método de disipación mínima, md-LDG. De esta forma se incluyen todas las variantes del método LDG. Sea $M$ la matriz de masa en la celda de referencia $[-1,1]$; para cualquier celda $I_{m}$ se tiene $M_{m}=\frac{h}{2} M$, luego

$$
\widehat{\Delta}_{h}=-\frac{2}{h} \widehat{A} \quad \text { donde } \widehat{A}=\widehat{B}_{1}^{*} M^{-1} \widehat{B}_{1}+\gamma \widehat{S} .
$$

Las propiedades del método se reducen al estudio del espectro de $\widehat{A}$, el cual no depende del incremento en tiempo $\tau$, pero sí del tamaño de la partición $h$, de la frecuencia $\omega$ y del grado del polinomio de aproximación.

Proposición 4.2. El símbolo $\widehat{S}$ del operador de estabilización $S(\cdot)$ es una matriz hermítica no negativa para toda aproximación de grado $p$.

Demostración. De acuerdo a la definición del término de estabilización de la ecuación (11) se tiene

$$
\widehat{S}=e^{-i \omega} E F_{-}+\left(I F_{-}+I F_{+}\right)+e^{i \omega} E F_{+},
$$

con

$$
E F_{ \pm}=\left[L_{i}( \pm 1) L_{j}(\mp 1)\right] \quad \text { y } \quad I F_{ \pm}=\left[L_{i}( \pm 1) L_{j}( \pm 1)\right]
$$

[Revista Integración, temas de matemáticas 
donde $\left.\left\{L_{i}(\cdot)\right)_{i=0}^{p}\right\}$ es una base de polinomios en la celda de referencia $[-1,1]$. Utilizando por ejemplo los polinomios de Legendre, el símbolo se reduce a

$$
\widehat{S}=\left[\begin{array}{rrrrr}
a & i b & a & i b & \ldots \\
-i b & c & -i b & c & \ldots \\
a & i b & a & i b & \ldots \\
-i b & c & -i b & c & \ldots \\
\vdots & \vdots & \vdots & \vdots & \ddots
\end{array}\right], \quad a=1-\cos (\omega), b=\sin (\omega), c=1+\cos (\omega) .
$$

Claramente $\widehat{S}$ es hermítica, y por la relación $a c=b^{2}$ se deduce que además es de rango 1 para todo $\omega \in[-\pi, \pi]$, por lo que $\widehat{S}$ posee dos valores propios: 0 de multiplicidad $p$ y otro de multiplicidad 1, y se puede verificar sin mayor dificultad que es positivo.

Proposición 4.3. Para todo $\gamma \geq 0$, los autovalores de la matriz $M^{-1} \widehat{A}$ son no negativos.

Demostración. Siendo $M$ la matriz de masa, esta es simétrica positiva definida. Considerando su descomposición de Cholesky, $M=L L^{T}$, se deduce que para todo eigenpar $(\lambda, x)$ de $\widehat{A}$ se tiene

$$
\lambda\|x\|^{2}=\langle\widehat{A} x, x\rangle=\left\langle\left(\widehat{B}_{1}^{*} L^{-T} L^{-1} \widehat{B}_{1}+\gamma \widehat{S}\right) x, x\right\rangle=\left\|L^{-1} \widehat{B}_{1} x\right\|^{2}+\gamma\langle\widehat{S} x, x\rangle \geq 0 .
$$

Por la Proposición 4.2 y el hecho de que $\gamma \geq 0$, se concluye que $\widehat{A}$ es hermítica no negativa; y a su vez, la matriz $L^{-T} \widehat{A} L^{-1}$ también lo es. El resultado se obtiene notando que las matrices $M^{-1} \widehat{A}$ y $L^{-T} \widehat{A} L^{-1}$ poseen el mismo espectro.

Proposición 4.4. Una condición necesaria y suficiente de estabilidad para el método FT$L D G$, aplicado a la ecuación del calor, es

$$
\tau \leq\left(\frac{1}{2 \lambda_{\max }}\right) \frac{h^{2}}{\sigma}
$$

donde $\lambda_{\max }$ es el autovalor de $M^{-1} \widehat{A}$ de mayor magnitud.

Demostración. Sean $I_{p}$ la matriz identidad de orden $p+1, \mathrm{y} \mu=\tau / h^{2}$. Escribiendo esta vez el parámetro de estabilidad en la forma $\eta=2 \frac{\sigma \gamma}{h}$ con $\gamma \geq 0$ constante, y aplicando la transformada discreta de Fourier, en su versión de sistemas, a las ecuaciones (13a) y (13b), el símbolo $\widehat{G}$ del esquema FT-LDG se reduce a

$$
\widehat{G}=I_{p}-4 \mu \sigma M^{-1} \widehat{A} .
$$

Por la no negatividad del espectro de $M^{-1} \widehat{A}$, Proposición 4.3, y como todo autovalor $\widehat{\lambda}$ de $\widehat{G}$ es de la forma $1-4 \mu \sigma \lambda$ con $\lambda$ autovalor de $M^{-1} \widehat{A}$, la hipótesis, desigualdad (14), equivale a tener $\rho(\widehat{G}) \leq 1$ (condición estricta de von Neumann).

La condición de von Neumann es además suficiente. Nótese que la matriz $G_{o}=I_{p}-$ $a \mu \sigma L^{-1} \widehat{A} L^{-T}$ es hermítica, por lo que $\rho\left(G_{o}\right)=\left\|G_{o}\right\|$; además, se tiene

$$
\widehat{G}=L^{-T} G_{o} L^{T} \quad \Longrightarrow \quad \rho(\widehat{G})=\rho\left(G_{o}\right) .
$$

Vol. 37, $\left.\mathrm{N}^{\circ} 2,2019\right]$ 
Luego, para todo $n \in \mathbb{N}$ se cumple

$$
\left\|\widehat{G}^{n}\right\| \leq\left\|L^{-T}\right\|\left\|L^{T}\right\|\left\|G_{o}^{n}\right\|=\left\|L^{-T}\right\|\left\|L^{T}\right\| \rho\left(G_{o}\right)^{n}=\left\|L^{-T}\right\|\left\|L^{T}\right\| \rho(\widehat{G})^{n} .
$$

Claramente, si la condición de von Neumann se cumple se tiene estabilidad, ecuación (7), con $C_{T}=\left\|L^{-T}\right\|\left\|L^{T}\right\|$, esta constante no depende $\omega, h$ y $\tau$.

En el Cuadro 1 se muestra el autovalor $\lambda_{\max }$ de mayor magnitud de la matriz $M^{-1} \widehat{A}$, para el método de disipación mínima, utilizando el flujo izquierdo. Ese valor fue obtenido numéricamente en MATLAB. Se observa un comportamiento asintótico de orden $\mathcal{O}\left((p+1)^{4}\right)$ para aproximaciones de grado $p$. Nótese que la condición de estabilidad para $p=0$ es la misma que la del método de diferencias finitas. Además se incluyó la constante $C F L(p)=1 /\left(2 \lambda_{\max }\right)$. Para el flujo derecho se obtuvo el mismo comportamiento, así como la misma condición CFL.

Obsérvese que la constante CFL (Courant-Friedrichs-Lewy) $1 /\left(2 \lambda_{\max }\right)$, para aproximaciones constantes, es decir $p=0$, es igual a la del esquema clásico de diferencias finitas. Por lo general se desea que esta constante sea lo más grande posible. Sin embargo, nótese que la condición de estabilidad es más restrictiva para aproximaciones de alto orden.

\begin{tabular}{cccc}
$p$ & $\lambda_{\max }$ & Tasa & $C F L(p)$ \\
\hline \hline 0 & $1.00000 \mathrm{e}+00$ & - & $5.00000000 \mathrm{e}-01$ \\
1 & $9.00000 \mathrm{e}+00$ & 3.17 & $5.55555556 \mathrm{e}-02$ \\
2 & $3.70646 \mathrm{e}+01$ & 3.49 & $1.34899708 \mathrm{e}-02$ \\
3 & $1.09727 \mathrm{e}+02$ & 3.77 & $4.55677642 \mathrm{e}-03$ \\
4 & $2.61323 \mathrm{e}+02$ & 3.89 & $1.91334185 \mathrm{e}-03$ \\
5 & $5.35669 \mathrm{e}+02$ & 3.94 & $9.33412975 \mathrm{e}-04$ \\
6 & $9.86259 \mathrm{e}+02$ & 3.96 & $5.06966074 \mathrm{e}-04$ \\
7 & $1.67631 \mathrm{e}+03$ & 3.97 & $2.98273467 \mathrm{e}-04$ \\
8 & $2.67878 \mathrm{e}+03$ & 3.98 & $1.86652121 \mathrm{e}-04$ \\
9 & $4.07633 \mathrm{e}+03$ & 3.98 & $1.22659257 \mathrm{e}-04$ \\
10 & $5.96138 \mathrm{e}+03$ & 3.99 & $8.38732492 \mathrm{e}-05$ \\
\hline
\end{tabular}

Cuadro 1. Comportamiento de $\lambda_{\max }$ y condición CFL como función del grado de aproximación para el método md-LDG.

En la Figura 1 se muestra el comportamiento de la condición CFL con respecto al término de estabilización $\gamma$ para el método LDG estabilizado. Se observa un comportamiento muy similar para los tres tipos de flujos y cualquier grado de aproximación. Para valores pequeños de $\gamma$ la CFL se comporta como la del método de disipación mínima, mientras que para valores grandes de $\gamma$ se observa una condición de estabilidad más severa.

En el Cuadro 2 se presenta la condición CFL para el término de estabilización $\gamma=1$ y los tres flujos considerados en este artículo. El valor elevado de la constante CFL para el flujo central muestra una leve ventaja de este en comparación con los flujos izquierdo y derecho. Esta se ilustra concretamente en la Figura 2, donde se muestra la aproximación obtenida para los flujos izquierdo y derecho, para un valor de $\mu$ más grande que el requerido por el criterio de estabilidad del Cuadro 2 para aproximaciones cuadráticas. Para obtener una aproximación correcta en el tiempo final $T=0,002$ estos flujos requieren 1957 pasos de

[Revista Integración, temas de matemáticas 


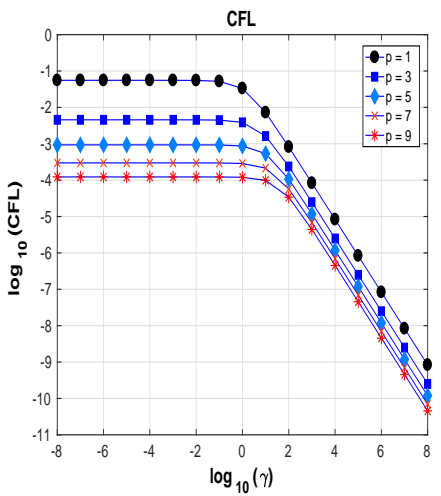

(a) Flujo izquierdo

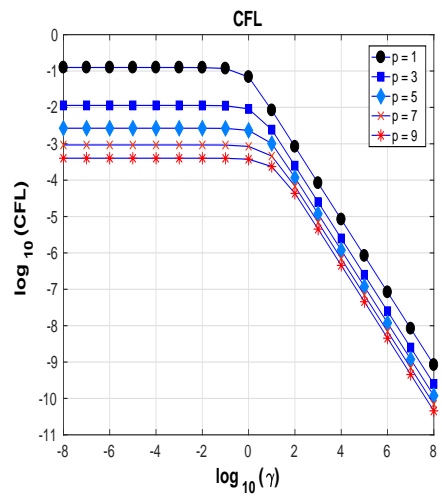

(b) Flujo central

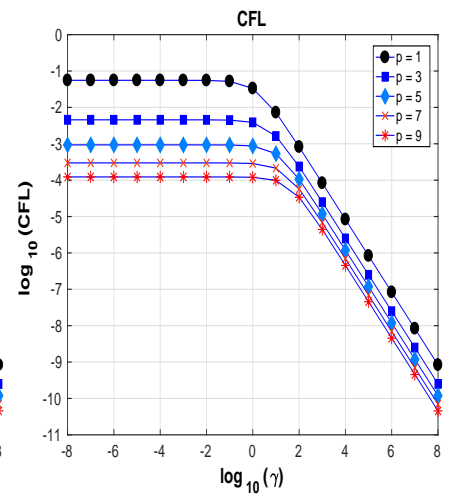

(c) Flujo derecho

Figura 1. Comportamiento de la CFL para el LDG estabilizado.

Flujos

\begin{tabular}{cccc}
\hline$p$ & Izquierdo & Central & Derecho \\
\hline \hline 1 & $3.33333333 \mathrm{e}-02$ & $\mathbf{6 . 8 6 9 1 5 5 8 1 e - 0 2}$ & $3.33333333 \mathrm{e}-02$ \\
2 & $1.02209934 \mathrm{e}-02$ & $\mathbf{2 . 2 2 9 3 9 1 3 4 e - 0 2}$ & $1.02209934 \mathrm{e}-02$ \\
3 & $3.88042871 \mathrm{e}-03$ & $\mathbf{9 . 0 9 4 9 5 4 8 6 e - 0 3}$ & $3.88042871 \mathrm{e}-03$ \\
4 & $1.72917277 \mathrm{e}-03$ & $\mathbf{4 . 3 4 9 6 2 7 9 9 e - 0 3}$ & $1.72917277 \mathrm{e}-03$ \\
5 & $8.71460746 \mathrm{e}-04$ & $\mathbf{2 . 3 3 3 1 8 7 2 3 e - 0 3}$ & $8.71460746 \mathrm{e}-04$ \\
6 & $4.82543550 \mathrm{e}-04$ & $\mathbf{1 . 3 6 2 0 3 4 4 8 e - 0 3}$ & $4.82543550 \mathrm{e}-04$ \\
7 & $2.87410305 \mathrm{e}-04$ & $\mathbf{8 . 4 6 9 5 7 9 9 1 e - 0 4}$ & $2.87410305 \mathrm{e}-04$ \\
8 & $1.81343639 \mathrm{e}-04$ & $\mathbf{5 . 5 3 4 0 6 9 0 8 e - 0 4}$ & $1.81343639 \mathrm{e}-04$ \\
9 & $1.19863144 \mathrm{e}-04$ & $\mathbf{3 . 7 6 3 8 7 2 9 4 e - 0 4}$ & $1.19863144 \mathrm{e}-04$ \\
10 & $8.23077586 \mathrm{e}-05$ & $\mathbf{2 . 6 4 6 2 8 9 2 5 e - 0 4}$ & $8.23077586 \mathrm{e}-05$ \\
\hline
\end{tabular}

Cuadro 2. CFL como función del grado de aproximación para el método LDG estabilizado con $\gamma=1$.

tiempo, mientras que para el flujo central solamente se necesitaron 897. Para este cálculo se consideró $\gamma=1$.

En la Figura 3 se comparan las aproximaciones obtenidas por el método md-LDG con polinomios cuadráticos y distintos valores de $\mu$, para el flujo izquierdo (subfiguras 3a-3c) y para el flujo derecho (subfiguras 3d-3f). Para este experimento $\sigma=1$, y el dominio $[0,1]$ se discretizó con una partición uniforme de tamaño $h=0,01$. Nótese la aparición de oscilaciones en las subfiguras $3 \mathrm{~b}, 3 \mathrm{c}, 3 \mathrm{e}$ y $3 \mathrm{f}$, debida a que los valores de $\mu$ utilizados no cumplen con la condición de estabilidad, $C F L(2) \approx 1,34899708 \times 10^{-2}$ del Cuadro 1 .

Con el propósito de mejorar la estabilización del método anterior, es decir, aumentar la constante CFL, se considera la siguiente modificación denotada por $\alpha$-FT-LDG, la cual consiste en utilizar la aproximación $u h^{n+\alpha}$ en el tiempo intermedio $t_{n+\alpha}=t_{n}+\alpha \tau$ :

$$
\begin{aligned}
\boldsymbol{q}_{m}^{n+\alpha} & =\sigma M^{-1} B_{1}\left(\boldsymbol{u}_{m-1}^{n}, \boldsymbol{u}_{m}^{n}, \boldsymbol{u}_{m+1}^{n}\right) \\
\boldsymbol{u}_{m}^{n+\alpha} & =\boldsymbol{u}_{m}^{n}-\alpha \tau M_{m}^{-1} B_{2}\left(\boldsymbol{q}_{m-1}^{n+\alpha}, \boldsymbol{q}_{m}^{n+\alpha}, \boldsymbol{q}_{m+1}^{n+\alpha}\right)-\alpha \tau \eta M_{m}^{-1} S\left(\boldsymbol{u}_{m-1}^{n}, \boldsymbol{u}_{m}^{n}, \boldsymbol{u}_{m+1}^{n}\right),
\end{aligned}
$$

Vol. 37, $\left.\mathrm{N}^{\circ} 2,2019\right]$ 


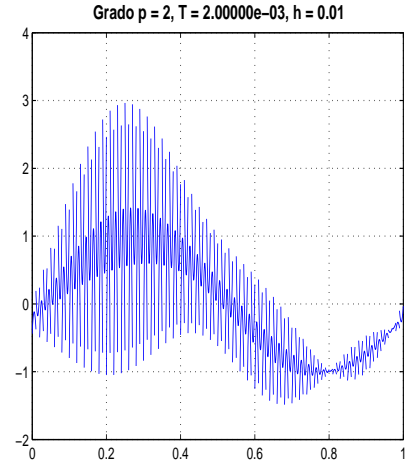

(a) $\mu \approx 1,3643875004 \times 10^{-2}$

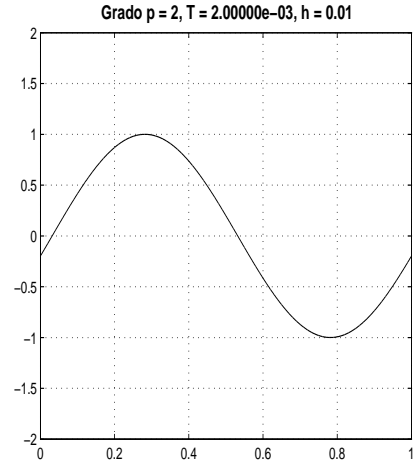

(b) $\mu \approx 2,2293913405 \times 10^{-2}$

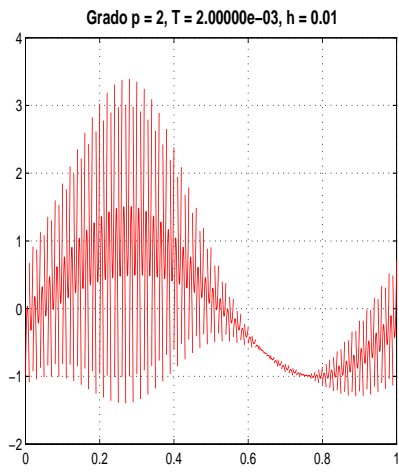

(c) $\mu \approx 1,3643875004 \times 10^{-2}$

Figura 2. FT-LDG flujos a) izquierdo, b) central y c) derecho.

$$
\begin{aligned}
\boldsymbol{q}_{m}^{n+1} & =\sigma M^{-1} B_{1}\left(\boldsymbol{u}_{m-1}^{n+\alpha}, \boldsymbol{u}_{m}^{n+\alpha}, \boldsymbol{u}_{m+1}^{n+\alpha}\right) \\
\boldsymbol{u}_{m}^{n+1} & =\boldsymbol{u}_{m}^{n}-\tau M_{m}^{-1} B_{2}\left(\boldsymbol{q}_{m-1}^{n+1}, \boldsymbol{q}_{m}^{n+1}, \boldsymbol{q}_{m+1}^{n+1}\right)-\tau \eta M_{m}^{-1} S\left(\boldsymbol{u}_{m-1}^{n+\alpha}, \boldsymbol{u}_{m}^{n+\alpha}, \boldsymbol{u}_{m+1}^{n+\alpha}\right) .
\end{aligned}
$$

Proposición 4.5. Una condición necesaria y suficiente de estabilidad para el esquema explícito $\alpha$-FT-LDG, aplicado a la ecuación del calor, es

$$
\tau \leq\left(\frac{1}{4 \alpha \lambda_{\max }}\right) \frac{h^{2}}{\sigma}, \quad \text { con } \quad \frac{1}{8} \leq \alpha
$$

donde $\lambda_{\max }$ es el mayor autovalor de la matriz $M^{-1} \widehat{A}$.

Demostración. El símbolo $\widehat{G}$ del método se escribe de la forma

$$
\widehat{G}=I_{p}-4 \mu \sigma M^{-1} \widehat{A}+16 \alpha(\mu \sigma)^{2}\left(M^{-1} \widehat{A}\right)^{2}
$$

y los autovalores de $\widehat{G}$ son de la forma $1-4 \mu \sigma \lambda+16 \alpha(\mu \sigma)^{2} \lambda^{2}$, donde $\lambda$ es un autovalor de $M^{-1} \widehat{A}$. La estabilidad del método se obtiene satisfaciendo la desigualdad

$$
\left|1-4 \mu \sigma \lambda+16 \alpha(\mu \sigma)^{2} \lambda^{2}\right| \leq 1
$$

lo cual equivale a satisfacer simultáneamente y para todo autovalor $\lambda$ de $M^{-1} \widehat{A}$ las desigualdades

$$
\begin{aligned}
& 0 \leq 1-2 \mu \sigma \lambda+8 \alpha(\mu \sigma)^{2} \lambda^{2} \\
& 0 \leq \mu \sigma \lambda(1-4 \alpha \mu \sigma \lambda)
\end{aligned}
$$

Observando que

$$
1-2 \mu \sigma \lambda+8 \alpha(\mu \sigma)^{2} \lambda^{2}=(1-\mu \sigma \lambda)^{2}+(8 \alpha-1)(\mu \sigma \lambda)^{2}
$$




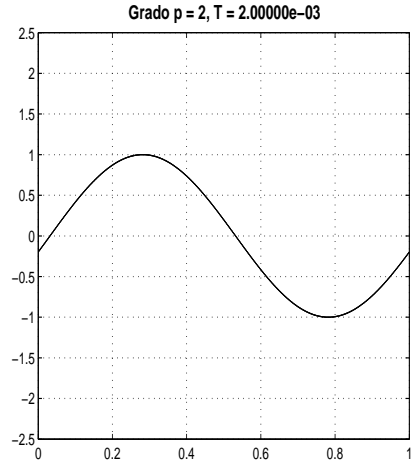

(a) $\mu=1,3490 \times 10^{-2}$

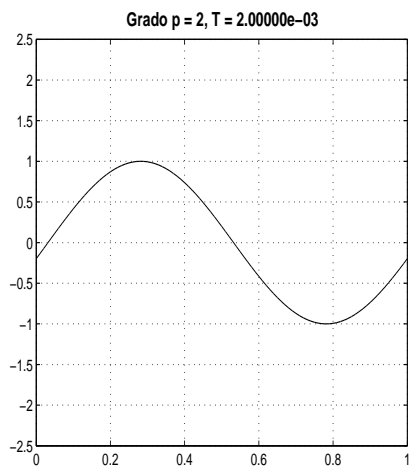

(d) $\mu=1,3485 \times 10^{-2}$

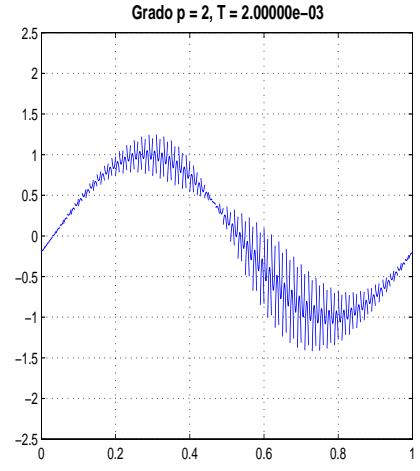

(b) $\mu=1,3660 \times 10^{-2}$

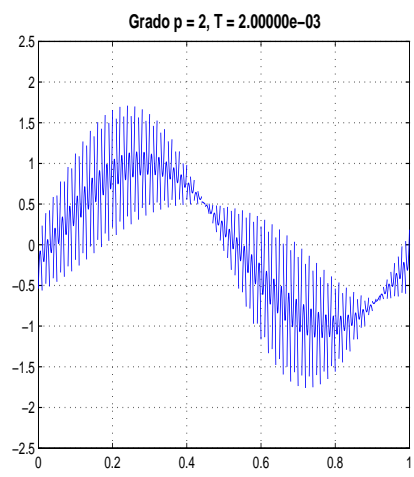

(e) $\mu=1,3660 \times 10^{-2}$

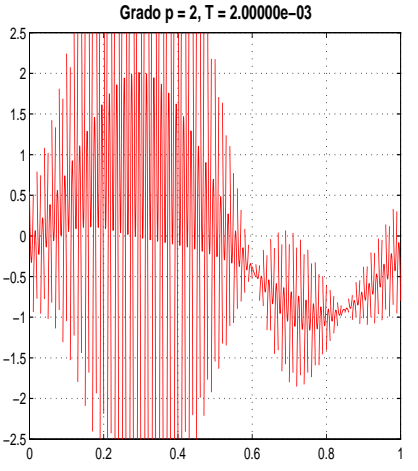

(c) $\mu=1,3670 \times 10^{-2}$

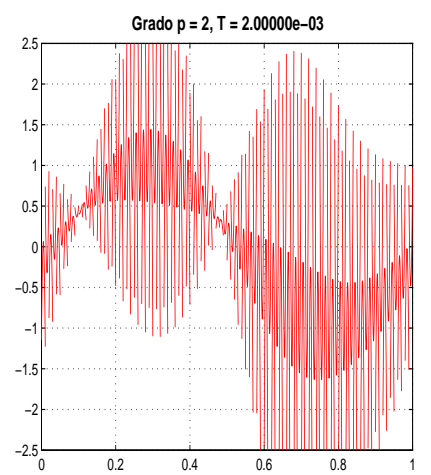

(f) $\mu=1,3670 \times 10^{-2}$

Figura 3. md-LDG con flujos izquierdo (3a-3c) y derecho (3d-3f), y aproximaciones cuadráticas.

la desigualdad (17) se verifica siempre que $8 \alpha-1 \geq 0$. Por otro lado, de acuerdo con la Proposición 4.3, los autovalores $\lambda$ son no negativos; y por la desigualdad (18) se tiene la condición

$$
0 \leq 1-4 \alpha \mu \sigma \lambda
$$

de suerte que se obtiene la condición estricta de von Neumann. Aplicando el mismo tipo de argumentos que en la prueba del método FT-LDG, se deduce que la condición es también suficiente.

Obsérvese que para $\alpha=1 / 8$ la constante CFL del esquema alcanza su valor máximo, el cual es cuatro veces mayor que el del esquema FT-LDG. Esta superioridad se ilustra en la Figura 4, donde se muestran las aproximaciones cúbicas obtenidas por este método, utilizando el flujo central con $\gamma=1$ y $\alpha_{o p t}=\frac{1}{8}$. Para la Figura 4a se utilizó el valor obtenido en la Proposición $4.5, \mu=4 \times C F L(3) \approx 3,6379819 \times 10^{-2}$, donde $C F L(3)$ corresponde al valor de CFL para aproximaciones cúbicas del Cuadro 2. Como era de

Vol. 37, $\left.\mathrm{N}^{\circ} 2,2019\right]$ 
esperar, la solución no presenta ningún tipo de oscilaciones, mientras que en la Figura 4b el cálculo se realizó con un valor de $\mu$ mayor.

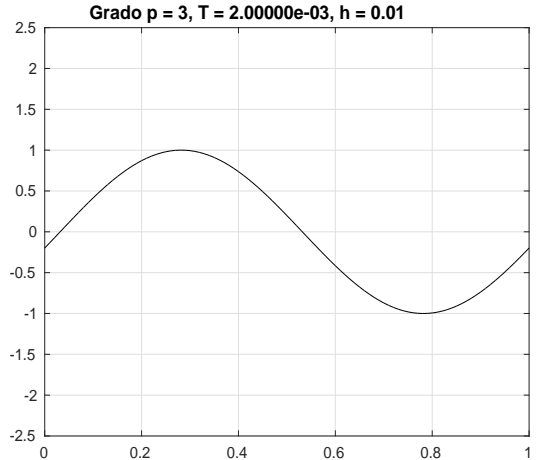

(a) $\mu=3,63798 \times 10^{-2}$

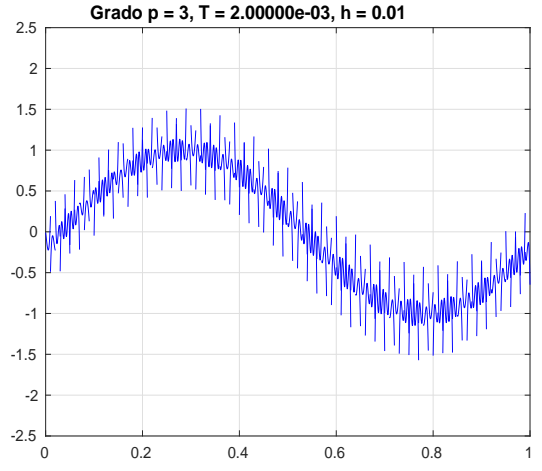

(b) $\mu=4,58280 \times 10^{-2}$

Figura 4. $\alpha$-FT-LDG con flujo central y grado $p=3$.

El uso de métodos explícitos, como los analizados anteriormente, se ve limitado debido a la severa restricción impuesta por la condición CFL. En la práctica es más conveniente el uso de métodos ímplicitos para problemas altamente difusivos y aproximaciones de alto orden. A continuación se analiza el método implícito denotado por BT-LDG aplicado a la ecuación de calor (ecuación (5)), el cual se expresa en la celda $I_{m}$ de la siguiente forma:

$$
\begin{aligned}
\boldsymbol{q}_{m}^{n+1} & =\sigma M_{m}^{-1} B_{1}\left(\boldsymbol{u}_{m-1}^{n+1}, \boldsymbol{u}_{m}^{n+1}, \boldsymbol{u}_{m+1}^{n+1}\right) \\
\boldsymbol{u}_{m}^{n+1} & =\boldsymbol{u}_{m}^{n}-\tau M_{m}^{-1} B_{2}\left(\boldsymbol{q}_{m-1}^{n+1}, \boldsymbol{q}_{m}^{n+1}, \boldsymbol{q}_{m+1}^{n+1}\right)-\tau \eta M_{m}^{-1} S\left(\boldsymbol{u}_{m-1}^{n+1}, \boldsymbol{u}_{m}^{n+1}, \boldsymbol{u}_{m+1}^{n+1}\right)
\end{aligned}
$$

Proposición 4.6. El método implícito BT-LDG es incondicionalmente estable para cualquier grado de aproximación.

Demostración. Realizando cálculos similares a los realizados en los esquemas anteriores se deduce que el símbolo del esquema implícito BT-LDG es

$$
\widehat{G}=(M+4 \mu \sigma \widehat{A})^{-1} M=\left(I_{p}+4 \mu \sigma M^{-1} \widehat{A}\right)^{-1}
$$

El resultado se obtiene observando que los autovalores $\widehat{\lambda}$ de $\widehat{G}$ son de la forma $(1+$ $4 \mu \sigma \lambda)^{-1}$, con $\lambda$ como autovalor de $M^{-1} \widehat{A}$; de acuerdo con la Proposición $4.3, \lambda \geq 0$, por lo que

$$
\widehat{\lambda}=\frac{1}{1+4 \mu \sigma \lambda} \leq 1
$$

independientemente del valor de $\mu$.

La estabilidad incondicional del método implícito BT-LDG se ilustra en la Figura 5, donde se muestra la aproximación con polinomios de grado 15 en el tiempo final $T=0,2$, para un incremento de tiempo $\tau=10^{5} \tau_{15}$, donde $\tau_{15}$ corresponde al incremento en tiempo 
requerido por la condición CFL del método FT-LDG para aproximaciones de grado 15 y $h=0,01, \tau_{15} \approx 6,4455 \times 10^{-9}$. Nótese que la aproximación obtenida no muestra oscilaciones y es cualitativamente correcta, a pesar de solo haber realizado 311 pasos de tiempo.

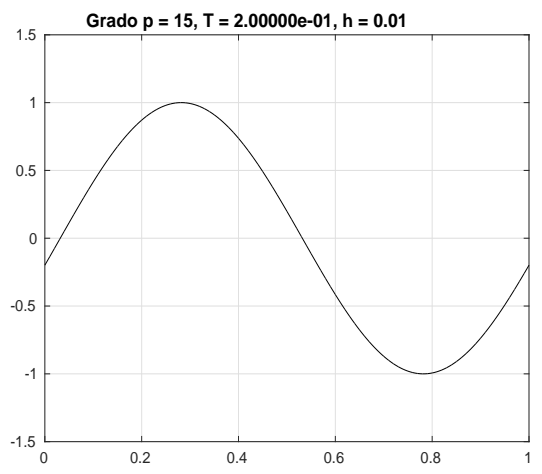

Figura 5. Aproximación con BT-LDG, flujo central, grado $p=15$ y $\tau=10^{5} \times \tau_{15}$.

\subsection{Ecuación de onda}

Otra de las ecuaciones fundamentales de la física es la ecuación de onda, la cual, por conveniencia, se escribirá de la siguiente manera:

$$
\begin{array}{r}
c^{-2} \boldsymbol{q}+\partial_{x} u=0, \\
u_{t t}+\partial_{x} \boldsymbol{q}=0,
\end{array}
$$

donde $c>0$. Asumiendo el parámetro de estabilidad $\eta$ constante, la formulación semidiscreta obtenida al aplicar la discretización espacial LDG puede escribirse localmente, en la celda $I_{m}$, como un sistema de ecuaciones diferenciales ordinarias:

$$
\begin{aligned}
c^{-2} M_{m} \boldsymbol{q}_{m} & =B_{1}\left(\boldsymbol{u}_{m-1}, \boldsymbol{u}_{m}, \boldsymbol{u}_{m+1}\right), \\
M_{m} \frac{d^{2} \boldsymbol{u}_{m}}{d t^{2}} & =-B_{2}\left(\boldsymbol{q}_{m-1}, \boldsymbol{q}_{m}, \boldsymbol{q}_{m+1}\right)-\eta S\left(\boldsymbol{u}_{m-1}, \boldsymbol{u}_{m}, \boldsymbol{u}_{m+1}\right) .
\end{aligned}
$$

Considerando la fórmula de diferenciación central para la segunda derivada en tiempo, se obtiene el método completamente discreto CT-LDG el cual se escribe en forma compacta como

$$
\begin{aligned}
M_{m} \boldsymbol{q}_{m}^{n+1} & =-c^{2} B_{1}\left(\boldsymbol{u}_{m-1}^{n}, \boldsymbol{u}_{m}^{n}, \boldsymbol{u}_{m+1}^{n}\right), \\
\frac{M_{m}}{\tau^{2}}\left(\boldsymbol{u}_{m}^{n+1}-2 \boldsymbol{u}_{m}^{n}+\boldsymbol{u}_{m}^{n-1}\right) & =-B_{2}\left(\boldsymbol{q}_{m-1}^{n+1}, \boldsymbol{q}_{m}^{n+1}, \boldsymbol{q}_{m+1}^{n+1}\right) \\
& -\eta S\left(\boldsymbol{u}_{m-1}^{n}, \boldsymbol{u}_{m}^{n}, \boldsymbol{u}_{m+1}^{n}\right) .
\end{aligned}
$$

A diferencia de la ecuación de calor, el operador de segundo orden en tiempo no permite una expresión explícita del símbolo, por lo que solamente se presentará una relación entre $\tau$ y $h$ que garantice la condición estricta de von Neumann.

Vol. 37, $\left.\mathrm{N}^{\circ} 2,2019\right]$ 
Proposición 4.7. Sea $\widehat{G}$ el símbolo del método CT-LDG aplicado a la ecuación de onda, ecuación (20); entonces la relación

$$
\tau \leq\left(\frac{1}{c \sqrt{\lambda_{\max }}}\right) h
$$

donde $\lambda_{\max }$ es el autovalor de $M^{-1} \widehat{A}$ de mayor magnitud, garantiza la condición estricta de von Neumann, es decir $\rho(\widehat{G}) \leq 1$.

Demostración. A diferencia de la ecuación del calor, ahora se espera una relación lineal entre $\tau$ y $h$. Sea $\mu=\tau / h$, y considérese el parámetro de estabilidad de la forma $\eta=2 \frac{c^{2} \gamma}{h}$ con $\gamma \geq 0$ constante. Por la hipótesis, se deduce que

$$
(\mu c)^{2} \lambda_{o} \leq 1, \quad \forall \lambda_{o} \in \sigma\left(M^{-1} \widehat{A}\right) .
$$

Nótese que el símbolo $\widehat{G}$ del esquema CT-LDG no se obtiene de manera explícita, pero satisface la ecuación matricial

$$
\widehat{G}^{2}+2\left(2(\mu c)^{2} M^{-1} \widehat{A}-I_{p}\right) \widehat{G}+I_{p}=0 .
$$

Por lo que, si $(\lambda, x)$ es un eigenpar de $\widehat{G}$, entonces satisface la igualdad

$$
(\lambda-1)^{2} x=-4(\mu c)^{2} \lambda M^{-1} \widehat{A} x,
$$

de modo que

$$
\lambda_{o}:=-\frac{(\lambda-1)^{2}}{4(\mu c)^{2} \lambda} \in \sigma\left(M^{-1} \widehat{A}\right) .
$$

Escribiendo $\lambda=a+i b$, de acuerdo con la Proposición 4.3, los autovalores de $M^{-1} \widehat{A}$ son no negativos, por lo que se tiene

$$
\text { i) } 0=\frac{b\left(1-|\lambda|^{2}\right)}{4(\mu c)^{2}|\lambda|^{2}} \quad \text { y } \quad \text { ii) } 0 \leq \frac{(2-\lambda)|\lambda|^{2}-\bar{\lambda}}{4(\mu c)^{2}|\lambda|^{2}} \text {. }
$$

Si $b=0, \lambda_{0}=-\frac{(a-1)^{2}}{4 \mu c^{2} a}, \lambda=a$ es una solución real de la ecuación cuadrática de coeficientes reales

$$
z^{2}+2\left(2(\mu c)^{2} \lambda_{0}-1\right) z+1=0 .
$$

Nótese que esta ecuación admite dos soluciones reales si, y solamente si,

$$
0<4\left(\left(2(\mu c)^{2} \lambda_{0}-1\right)^{2}-4=16(\mu c)^{2} \lambda_{o}\left((\mu c)^{2} \lambda_{0}-1\right)\right),
$$

lo cual contradice la desigualdad de la hipótesis (24). Para el caso límite, $(\mu c)^{2} \lambda_{0}=1$, no hay problema, ya que $\lambda=-1,(|\lambda|=1)$. Por lo tanto, para todo $\lambda \in \sigma(\widehat{G}),|\lambda|^{2}=1$. Además, $\lambda_{0}=\frac{1-a}{2(\mu c)^{2}} \Longleftrightarrow a=1-2 \lambda_{0}(\mu c)^{2}$, de donde

$$
0 \leq b^{2}=1-a^{2}=1-\left(1-2 \lambda_{0}(\mu c)^{2}\right)^{2}=4 \lambda_{0}(\mu c)^{2}\left(1-\lambda_{0}(\mu c)^{2}\right) .
$$

Por la hipótesis, esta desigualdad se cumple para todo $\lambda_{o}$. 
Comentario 1. Del cuadro 1 tenemos que $\lambda_{\text {máx }}(0)=1$ para el método md-LDG; en este caso, la condición CFL del método CT-LDG coincide con la del método de diferencias finitas, $\tau \leq \frac{h}{c}$.

Comentario 2. La ecuación (26) posee dos soluciones reales cuando $1<(\mu c)^{2} \lambda_{0}$. Nótese que en este caso una de las soluciones, $\lambda=\omega-\sqrt{\omega^{2}-1}<-1$, donde $\omega=1-(\mu c)^{2} \lambda_{0}$, por lo que el método sería inestable. En el siguiente ejemplo se ilustra la inestabilidad del método al violar la condición (23). Para ello se considera el siguiente problema:

$$
\begin{aligned}
u_{t t} & =u_{x x}, & & (x, t) \in(-L, L) \times(0, T], \\
u(x, 0) & =e^{-x^{2} / \sigma^{2}}, & & x \in(-10,10), \\
u_{t}(x, 0) & =0, & & x \in(-10,10), \\
u(-10, t) & =u(10, t), & & t \in(0, T],
\end{aligned}
$$

cuya solución exacta está dada por $u_{\sigma}(x, t)=\frac{1}{2}\left(e^{-\left(\frac{x+t}{\sigma}\right)^{2}}+e^{-\left(\frac{x-t}{\sigma}\right)^{2}}\right)$. El primer paso del método CT-LDG se realiza de forma análoga que para el método de diferencias finitas, denotando por $U_{h}^{-1}$ un paso auxiliar:

$$
\frac{U_{h}^{1}-U_{h}^{-1}}{2 \tau}=\Pi_{h, 0},
$$

donde $\Pi_{h, 0}$ es la proyección $L_{2}$ de la condición inicial $u_{t}(x, 0)$; luego, utilizando esta aproximación de $U_{h}^{-1}$, se aplica el método CT-LDG para encontrar $U_{h}^{1}$. En la Figura 6 se muestran las aproximaciones obtenidas por el método md-LDG con polinomios cuadráticos y distintos valores de $\mu$, para el flujo derecho. El dominio se discretizó con una partición uniforme de tamaño $h=0,2$. Nótese la aparición de oscilaciones en las figuras $6 \mathrm{~b}, 6 \mathrm{c}$, debida a que los valores de $\mu$ utilizados no cumplen con la condición de estabilidad, $C F L(2) \approx 1,64255720 \times 10^{-1}$.

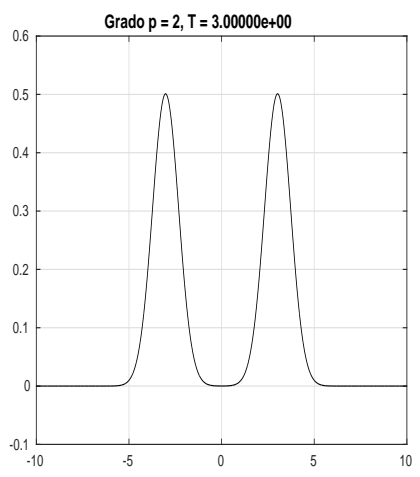

(a) $\mu=1,6425 \times 10^{-1}$

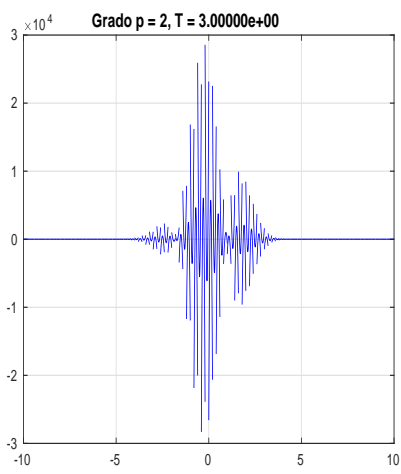

(b) $\mu=1,7025 \times 10^{-1}$

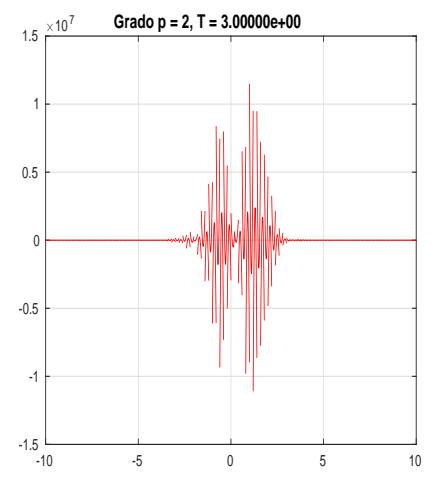

(c) $\mu=1,7225 \times 10^{-1}$

Figura 6. md-LDG con flujo derecho y grado $p=2$. 


\subsection{Ecuación lineal de Schrödinger}

Realizando un procedimiento análogo al de la Proposición (4.4) se puede demostrar, sin mayor dificultad, que el símbolo $\widehat{G}$ del esquema FT-LDG aplicado a la ecuación lineal de Schrödinger, ecuación (6), es

$$
\widehat{G}=I_{p}-4 i \mu \sigma M^{-1} \widehat{A} .
$$

Por lo tanto, el método FT-LDG aplicado a dicha ecuación corre la misma suerte que el de la discretización espacial basada en diferencias finitas, es decir, se tiene el siguiente resultado:

Proposición 4.8. El método explícito FT-LDG aplicado a la ecuación lineal de Schrödinger es incondicionalmente inestable para cualquier grado de aproximación.

Demostración. Nótese que todo autovalor $\widehat{\lambda}$ de $\widehat{G}$ es de la forma $1-4 i \mu \sigma \lambda$, con $\lambda$ como autovalor de $M^{-1} \widehat{A}$. Por la no negatividad del espectro de $M^{-1} \widehat{A}$, Proposición (4.3), se tiene

$$
|\widehat{\lambda}|=1+4 \mu \sigma \lambda \geq 1
$$

por lo que el método FT-LDG aplicado a la ecuación lineal de Schrödinger es inestable.

En [4] Castillo y Gómez presentaron un análisis de estabilidad y un estudio numérico de la condición CFL para el esquema explícito "pídola" en combinación con la discretización espacial LDG aplicado a la ecuación lineal de Schrödinger fraccionaria. En dicha ecuación se consideró la derivada fraccionaria de Riesz, de orden $1<\alpha \leq 2$, como operador diferencial.

\section{Conclusiones}

En este trabajo se ha ilustrado el uso del análisis de estabilidad de von Neumann para obtener condiciones suficientes y necesarias de estabilidad numérica para varias discretizaciones explícitas en tiempo, en combinación con la discretización espacial LDG para el operador de difusión. Se mostró, numéricamente, que el comportamiento asintótico de la constante CFL en función del grado de aproximación $p$ decrece de orden $\mathcal{O}\left((p+1)^{-4}\right)$ para la ecuación del calor, y de orden $\mathcal{O}\left((p+1)^{-2}\right)$ para la ecuación de onda. El uso de una discretización temporal explícita para problemas difusivos, en combinación con la discretización espacial LDG, se ve muy limitado debido a la severa restricción en el incremento en tiempo de orden $\mathcal{O}\left(h^{2}\right)$. La superioridad de discretizaciones temporales implícitas se ilustró mediante un ejemplo en el cual se permite un paso de tiempo mucho más grande que el de una explícita. Sin embargo, el estudio de la CFL es relevante para aquellos investigadores que prefieren el uso de técnicas explícitas en tiempo.

En general, el análisis muestra un comportamiento similar al de discretizaciones espaciales basadas en diferencias finitas, tanto para la ecuación de calor como para la ecuación de onda y la de Schrödinger.

Agradecimiento. Agradecemos a los evaluadores anónimos por sus valiosas sugerencias y comentarios, los cuales contribuyeron a mejorar la presentación de este artículo.

[Revista Integración, temas de matemáticas 


\section{Referencias}

[1] Castillo P., "Stencil reduction algorithms for the Local Discontinuous Galerkin method", Internat. J. Numer. Methods Engrg. 81 (2010), No. 12, 1475-1491.

[2] Castillo P., Cockburn B., Perugia I. and Schötzau D., "An a priori error analysis of the Local Discontinuous Galerkin method for elliptic problems", SIAM J. Num. Anal. 38 (2000), No. $5,1676-1706$.

[3] Castillo P., Cockburn B., Schötzau D. and Schwab Ch., "An optimal a priori error estimate for the $h p$-version of the Local Discontinuous Galerkin method for convection-diffusion problems", Math. Comp. 71 (2001), No. 238, 455-478.

[4] Castillo P. and Gómez S., "On the conservation of fractional nonlinear Schrödinger equation's invariants by the Local Discontinuous Galerkin method", J. Sci. Comput. 77 (2018), No. 3, 1444-1467.

[5] Charney J.G., Fjörtoft R. and Von Neumann J., "Numerical integration of the barotropic vorticity equation", Tellus 2 (1950), No. 4, 237-254.

[6] Cockburn B. and Dawson C., "Some extensions of the Local Discontinuous Galerkin method for convection diffusion equations in multidimensions", In Proceedings of the Conference on the Mathematics of Finite Elements and Applications: MAFELAP X( ed. Whiteman J. R.), Elsevier (2000), 225-238.

[7] Cockburn B. and Dong B., "An analysis of the minimal dissipation Local Discontinuous Galerkin method for convection diffusion problems", SIAM J. Sci. Comput. 32 (2007), No. $2,233-262$.

[8] Cockburn B. and Shu C.W., "The Local Discontinuous Galerkin method for time-dependent convection-diffusion systems", SIAM J. Num. Anal. 35 (1998), No. 6, 2440-2463.

[9] Gustafsson B., Kreiss H.O. and Oliger J., Time dependent problems and difference methods, John Wiley \& Sons, Inc., New York, 1995.

[10] Kubatko E.J., Dawson C. and Westerink J.J., "Time step restrictions for Runge-Kutta discontinuous Galerkin methods on triangular grids", J. Comput. Phys. 227 (2008), No. 23, 9697-9710.

[11] Kubatko E.J., Westerink J.J. and Dawson C., "Semi discrete discontinuous Galerkin methods and stage-exceeding-order, strong-stability-preserving Runge-Kutta time discretizations", J. Comput. Phys. 222 (2007), No. 2, 832-848.

[12] Perugia I. and Schötzau D., "An $h p$-analysis of the Local Discontinuous Galerkin method for diffusion problems", J. Scientific Computing. 17 (2002), No.1-4, 561-571.

[13] Strikwerda J.C., Finite difference schemes and partial differential equations, SIAM, Philadelphia, 2004.

[14] Toulorge T. and Desmet W., "CFL conditions for Runge-Kutta discontinuous Galerkin methods on triangular grids", J. Comput. Phys. 230 (2011), No. 12, 4657-4678.

Vol. 37, $\left.\mathrm{N}^{\circ} 2,2019\right]$ 